\title{
Determining the cast core inclination in absence of guidance from natural teeth - a distinctive clinical technique
}

\author{
Authors \\ Khurshid A Mattoo ${ }^{1^{*}}$, Areej Darraj ${ }^{2}$, Gupta $\mathbf{I}^{3}$ \\ ${ }^{1}$ Assistant Professor, Department of Prosthetic Dental Science, Jazan University, (KSA) \\ ${ }^{2}$ Student (UG), Comprehensive care clinic, Jazan University, (KSA) \\ ${ }^{3}$ Assistant Professor, Department of Conservative Dentistry and Endodontics, Jazan University, (KSA) \\ *Corresponding Author \\ Khurshid A Mattoo \\ Email:drkamattoo@rediffmail.com
}

\begin{abstract}
Among the most difficult cases of full mouth occlusal rehabilitation, very few fall in the realm where there is left no guidance for restorative build up either from adjacent teeth or from opposing teeth. In such cases, one has to utilize basic knowledge and understanding of complete denture fabrication and innovate to rehabilitate the dentition successfully. An adult female complaining of non functional teeth reported to comprehensive clinic for restoration of her entire dentition. Medical history disclosed a case of active rheumatoid arthritis, while clinical examination revealed grossly decayed natural dentition. The extent of damage was severe with ninety percent of the coronal tooth structure in entire visible dentition destroyed by caries. Endodontic treatment and crown lengthening procedures were compulsory to accomplish the prosthodontic objectives. Complexity of rehabilitation lied in determining the inclination of cast core in relation to the most aesthetic placement of the crowns which had to be fabricated after the cementation of cores. Principles of complete denture rehabilitation were implied by using a modified trial denture base occlusal rim, on which jaw relation was first determined. Artificial teeth were modified and arranged following which a trial was done. This trial denture was then used as a template to give inclinations to the underlying cores during laboratory procedures. Single crowns, a six unit partial denture and a cast partial denture were prosthetic components of rehabilitation. The patient was highly satisfied with the functional outcome and continues to be to on a follow up protocol.

Keywords: dowel core, complete denture, crown lengthening, endodontic, rheumatoid arthritis.
\end{abstract}

\section{Introduction}

Utilizing the root of a tooth for retention of the crown is a concept that dates back to the eighteenth century, when Fauchard inserted wooden dowels to aid in crown retention. ${ }^{1}$ Since then the dowel core has evolved in terms of design, material used, convenience, preparation features and clinical outcome. The present cast dowel core evolved from its ancestral One - piece dowel crown also called as a Richmond crown (Introduced in 1878). ${ }^{2}$ Use of cast post and cores have now become a routine method of restoration, mainly due to the advent in scientific endodontic advent therapy.

Cast post cores are the first restorative choice for anterior teeth if the teeth have moderate to severe destruction. Posterior teeth perform satisfactorily with prefabricated direct cores. Fabrication of cast post cores is done by two methods: Direct and 
indirect. $^{3,4}$ The pattern of the cast post core is prepared on the patient in direct technique while the same built up when done on a cast in the laboratory is termed indirect technique. When natural teeth are present adjacent to the tooth being restored or opposite to the tooth being restored, fabrication of the core pattern is relatively easy in term of of its length, width, thickness and labiolingual axial inclination. The problem lies in grossly decayed natural dentition where there is no clinical guidance either to the clinician or to the laboratory technician. This article presents a case report of an adult female whose entire natural dentition was grossly decayed, and there were no natural teeth that could guide adequate length, width, thickness and inclination of the core cast. In order to reduce clinical and laboratory errors, principles of completed denture fabrication were utilised which gave rise to a novel technique of cast core fabrication in such situations. Troubleshooting with the technique is also discussed.

\section{Case Report}

A youthful female patient, in her late twenties was brought by an undergraduate female student as her comprehensive care patient during her undergraduate course. Despite the case being rejected by many of her multispeciality staff members, the student was determined to treat the patient with much fear and apprehension. The reason being that the patient had severe destruction of natural dentition which was compounded by her underlying systemic medical condition of active and under medication rheumatoid arthritis (RA) (since one year). The medication regimen of methotrexate, Celebrex and Humira increased the challenges of rehabilitation because extractions, crown lengthening and endodontic procedures were unavoidable.

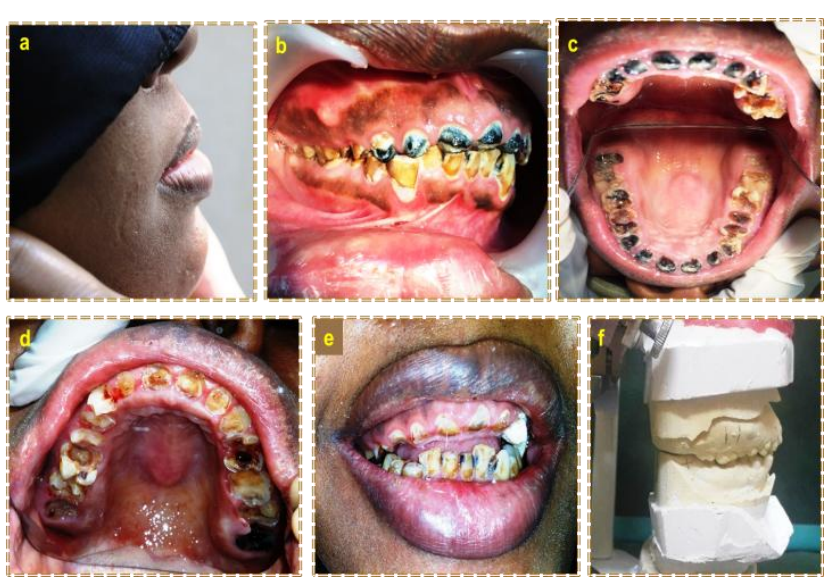

Figure 1: Extra oral facial profile (a), Intra oral view showing gross destruction of natural dentition as a result of caries (b,c), diagnostic caries removal followed by temporary restorations (d,e), mounted diagnostic cast on a semi adjustable articulator.

To make the matters worse temperomandibular joint involvement, fatigue and xerosis were evident in the patient before and after her diagnosis of RA. Hair loss, frequent infections, infrequent tooth brushing, ignorant self care and patients low self esteem were other features revealed during thorough history taking. Extra oral examination relevant features included thick lips (Fig.1a), TMJ crepitation, unaesthetic hair loss, broad smile with high lip line and a high low lip line.

The intra oral situation was grossly mutilated natural dentition to the level of gingiva in the anterior and mid posterior region (Fig.1b,c), with vertical dimensions of occlusion maintained by left premolar teeth although both had worn down. To make a clear clinical diagnosis all the caries was removed in the first appointment (Fig.1d,e), following which preliminary impression were made with irreversible hydrocolloid and diagnostic casts were mounted on a semi adjustable articulator (Whip Mix; Elite Dental Services,Orlando,Fla) programmed at this stage with centric interocclusal record while average values were taken for protrusive and lateral movements (Fig 1.f). Full mouth periapicals and orthopantomograph were taken that demonstrated root stumps, periapical lesions, periodontitis and pulpal involvement due to caries (Fig 2.a). Medical investigations included complete blood count, electrolytes and BUN (blood 
urea nitrogen), electrocardiogram, blood gases and liver function tests. Treatment plan presented to the patient included a full mouth rehabilitation using fixed and removable partial dentures after compulsory extraction of ten teeth which would be followed by endodontic treatment of tooth no 13 , 14, 15,16,17, 23, 24,25, 27 and 46 and crown lengthening of maxillary and mandibular arches on both labial and lingual sides. After consultation and approval from the rheumalogist, an informed consent of the patient was taken.

Immunosuppressant drugs were stopped two weeks prior to surgical procedures and all surgical procedures (extraction and crown lengthening) were completed within a space of two weeks under an antibiotic coverage (Amoxicillin $2 \mathrm{~g}$ in divided dose for ten days) and prophylactic antibiotic coverage (2 g orally 1 hour before major surgical procedure) (Fig 2 b,c,d).

Prosthetic rehabilitation started after resuming of the current medication. At this stage, the natural, healthy tooth structure was isolated in the oral cavity after which impressions were made with irreversible hydrocolloid for both arches. A surgical cast was then made from the impression by pouring die stone in it which was then corrected for any irregularities. A modified trial denture base was formed in which all maxillary natural teeth were relieved through perforation within the denture base (Fig.3a). Occlusal rims were then prepared on this modified denture base and jaw relations were recorded like that done for complete denture fabrication with particular emphasis on the profile contour of the occlusal rims and the visibility of the rims that would be aesthetically pleasing (Fig.3b). Artificial teeth were then modified by removing the lingual surfaces of all the anterior teeth leaving only labial surfaces and then arranged on the occlusal rims. Multiple anterior trials were then performed (Fig.3c, d) till aesthetics were satisfactory. Once aesthetics were appropriated the wax around the teeth was replaced with self cure denture acrylic so that the trial denture would act as guiding splint both in the laboratory and in the clinic to verify/correct the inclination of the core cast. When placing the trial denture in the patient's mouth, the underlying teeth along with their respective post space was clearly visible (Fig.3e, f).

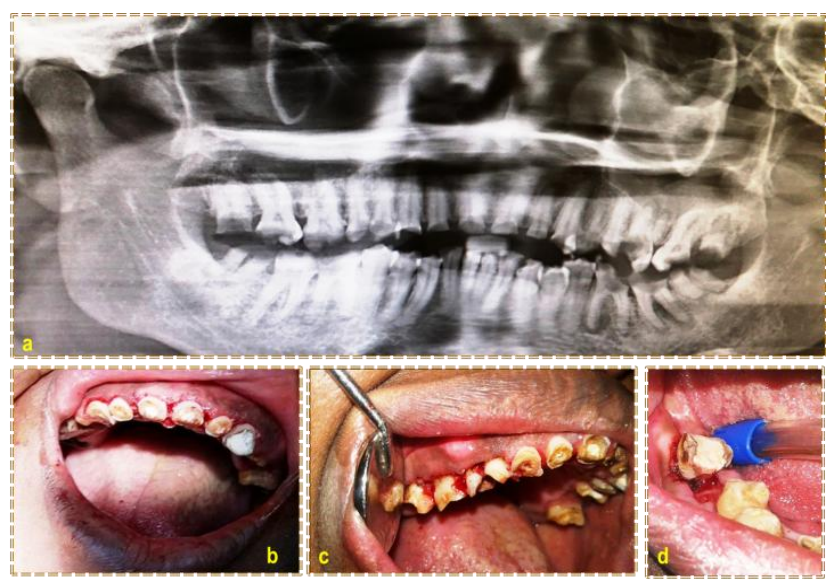

Figure 2: Orthopantomograph showing relationship of pulp to existing overlying natural tooth structure (a), crown lengthening procedure (b,c,d)

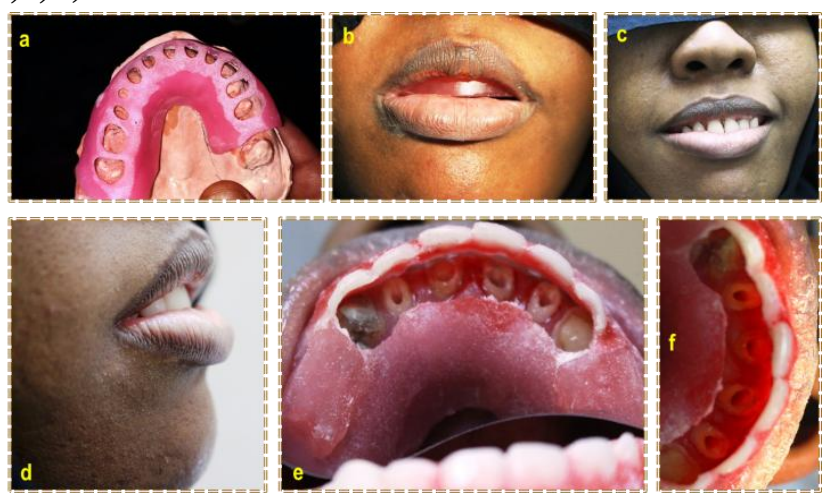

Figure 3: Acrylic guiding splint (a), establishing profile contour on a wax occlusal rim (b), artificial teeth arranged for trial (c,d), creation of a window to expose the underlying natural teeth and verify the axial inclination of the core (e,f)

All maxillary anterior teeth received cast post core while maxillary premolars were restored using prefabricated fibre posts (Rely X fibre post 3M ESPE) (Fig. 4a). Mandibular arch was restored with a six unit fixed partial denture anteriorly and a three unit fixed partial denture posteriorly (Fig.4b). Few endodontic failures in the mandibular anterior region were a part of the modified treatment plan. After cementation of all cast post core foundation restorations (Fig.4c), temporization was done using CADCAM (CEREC-AC Sirona) (Fig.4d). The patient was then asked to use the temporary for a 
period of 12-16 weeks to verify the effects of newly established anterior guidance and vertical dimensions. After minor adjustments of the temporary crowns, definitive restorations were fabricated by doing metal trial following which a pick up impression was made for each arch (Fig. 4e). Maxillary and mandibular anteriors were restored based on the principles of Pankey Mann Schulyer philosophy while following Dawsons quadrant arch approach (Fig.5a, b). A cast partial denture was fabricated along with respective surveyed crowns. A mutually protected occlusion was provided with stable and coordinated occlusal contacts (Fig.5c). Home care instructions were demonstrated to the patient regarding oral hygiene maintenance and patient was put on a regular follow up for one year. The patient was extremely satisfied with her occlusal rehabilitation with improved aesthetics, phonetics and masticatory function (Fig.5d,e).

\section{Discussion}

The case presented in this article is that of an adult female who was previously diagnosed with rheumatoid arthritis which in her case progressed chronically. The condition is a systemic disease which is characterized pathologically by widespread changes in various tissues and organs. Surgical intervention in such cases should be properly scheduled to avoid the postsurgical infections.

Endodontic treatment of a vast number of teeth,
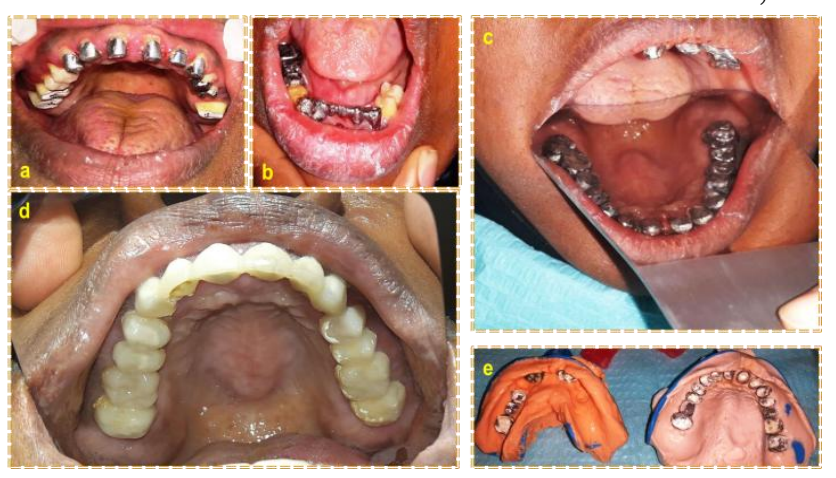

Figure 4: Cast post core cemented in maxillary teeth (a), fixed the mandibular anterior regionandibular anterior region (b), posterior maxillary metal trail (c), CADCAM temporary restorations (d) and pick up impressions with metal trial crowns (e)

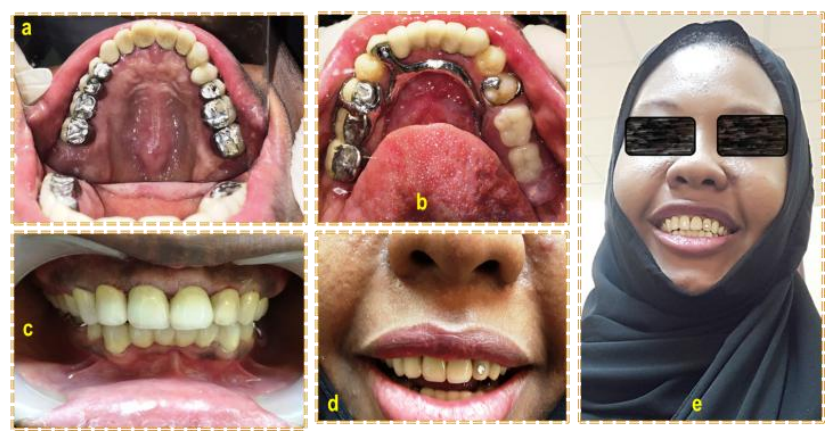

Figure 5: Completed rehabilitation of maxillary teeth with individual crowns (a), mandibular arch (b), frontal view of completed occlusal rehabilitation $(c, d)$ and extra oral view of the patient after 3 months follow up(e)

While fabricating a dowel core pattern either in the mouth directly or on the cast indirectly requires presence of clinical guide which adjacent or opposing teeth provide either to the clinician or to the laboratory technician. In this case, while crown forms of all maxillary anterior teeth were missing, most of the mandibular anteriors were extracted or decayed due to caries. Such situations are rarely encountered in daily clinical practice. To be best of our knowledge, there is no technique in the literature that mentions what the clinician or the laboratory technician must do for such cases. Mostly, aesthetics in such cases are done by trial and error method which is time consuming. One way is to fabricate the core, according to the post space, but that only describes the labiolingual thickness of the core. If individual patterns are made directly in the mouth, even then there is no way to determine their relation with regard to the final restoration. Muneera et.al described a technique to determine the aesthetic inclination of the cast post core, in which they prepared a putty index after making individual temporary and use the same putty index to see whether the cast core are correctly inclined or not. ${ }^{8}$ However, the advantage with the technique mentioned in this article is that the modified trial denture base can be used during laboratory pattern to build up and also during the trial of the core cast. Moreover the technique mentioned in this article can be used when none of the natural teeth can guide the build up while that described by Muneera et al is when some of the natural teeth are present. 
Troubleshooting: This technique is based on the principles of complete denture rehabilitation where there is no guidance present to place natural tooth. In such cases the position taken is ideal rather than what the position was on that particular patient. So the patient may feel differently between the artificial teeth and previous natural teeth. Natural tooth structure that is present may not be totally absent and some portion of it may be above the gingiva in which case provision of relief becomes critical. Because the clinician knows the clinical picture of the patient's mouth, it is advised that the clinician may provide relief himself or direct the technician to do accordingly. There is little or no wax present behind the labial facing of artificial teeth while verifying the cast core inclination on the patient. This renders the trial procedure cumbersome and easily distorted by patient's lips. Therefore the use of modified trial denture should be first used in the laboratory to allow a technician to determine the inclination of cast core. Once the laboratory procedure is over, the technician should be asked to replace wax with self cure denture resin which makes it difficult to get distorted. In order to avoid refined of the cast core at chairside, it is advisable that the patterns be tried in the mouth before casting them.

\section{Conclusion}

Determining cast core inclination relative to the final position of the teeth, which is not present is important clinical procedure that saves the clinician from much trial and error procedures and obvious embarrassment in front of the patient. The technique is based on sound clinical principles of complete denture rehabilitation and gave us excellent results.

\section{Acknowledgement}

The authors would like to acknowledge the determination of the undergraduate student who, despite being rejected the case by many staff pursued in the interest of the patient well being.

\section{References}

1. Fauchard P. The surgeon dentist or treatise on the teeth, vol. 2. Translated from the 2nd ed. 1746 by Londsay L. London: Butterworth and Company

2. Demas NC. Direct impression for cast Richmond crown using acetate crown forms. Dental Dig 1957;63:258-9.

3. Barker BC. Restoration of non-vital teeth with crowns. Aust Dent J 1963;8:191-200.

4. Sall HD. Restorative techniques for endodontically treated teeth. Dent Surv 1977;53:45-7.

5. Mann AW, Pankey LD. Oral rehabilitation: part I. Use of the P-M instrument in treatment planning and in restoring lower posterior teeth. J Prosthet Dent. 1960;10:135-150

6. Dawson PE. Evaluation, diagnosis and treatment of occlusal problems. St Louis: CV Mosby Co; 1974. pp. 148-9

7. Darraj A, Mattoo KA, Nazish M. Scheduling multiple surgical procedures in immunocompromised rheumatoid patient on immunosuppressant regimen. Journal of Medical science and clinical research. 2017;5(8):26828-33

8. Muneera RAG, Mattoo KA, Youseef AM. A novel approach to determine the aesthetic inclination of cast post core - case report. Annals of international medical and dental research, 2017;3(6):14-17 